Han-Liang Huang* and Yuting Guo

\title{
An Improved Correlation Coefficient of Intuitionistic Fuzzy Sets
}

DOI 10.1515/jisys-2017-0094

Received March 12, 2017; previously published online July 6, 2017.

\begin{abstract}
The intuitionistic fuzzy set is a useful tool to deal with vagueness and uncertainty. Correlation coefficient of the intuitionistic fuzzy sets is an important measure in intuitionistic fuzzy set theory and has great practical potential in a variety of areas, such as decision making, medical diagnosis, pattern recognition, etc. In this paper, an improved correlation coefficient of the intuitionistic fuzzy sets is defined, and it can overcome some drawbacks of the existing ones. The properties of this correlation coefficient are discussed. Then, the generalization of the coefficient of interval-valued intuitionistic fuzzy sets is also introduced. Finally, two examples about the application of the proposed correlation coefficient of the intuitionistic fuzzy sets in medical diagnosis and clustering are shown to illustrate the advantages over the existing methods.
\end{abstract}

Keywords: Correlation coefficient, intuitionistic fuzzy sets, interval-valued intuitionistic fuzzy sets, medical diagnosis, clustering.

2010 AMS Classification: 03E72.

\section{Introduction}

The concept of the intuitionistic fuzzy set (IFS) proposed by Atanassov [1] is a generalization of the fuzzy set [26]. One of the characterization of the IFS is that it assigns to each element a membership degree and a non-membership degree rather than the membership degree only. The IFS is more flexible and practical for dealing with vagueness and uncertainty than the ordinary fuzzy set in many real situations. Then, Atanassov and Gargov [2] further generalized the IFS to an interval-valued intuitionistic fuzzy set (IVIFS) in which the values of its membership degree and non-membership degree are intervals. The IFS is closely related to other generalized fuzzy sets such as $L$-fuzzy sets [5, 7, 18, 23] and interval-valued fuzzy sets (IVFSs) [13, 15, 27, 29], and it has been widely applied in medical diagnosis $[8,14,30]$, decision making $[20,24]$, clustering $[3,9,25]$ and pattern recognition $[4,6,12]$.

The measurement of correlation between two IFSs plays an important role in IFS theory, and it is always formulated by correlation coefficient. Therefore, how to define an effective correlation coefficient formula is an interesting research topic. Many scholars have paid great attention to this issue and obtained many useful results. For example, Hung and Wu [11] proposed a method to calculate the correlation coefficient of IFSs by means of "centroid", which reflected not only the correlation between IFSs but also their positive or negative correlation. Moreover, they extended the "centroid" method to IVIFSs. Hung [10] investigated the correlation measure of IFSs from the perspective of statistics. Xu developed a correlation coefficient formula [20] of IFSs, which was generalized to the IVIFSs. Then, Xu proposed a decision-making method in medical diagnosis under the IFS environment. He also proposed another correlation coefficient formula in Ref. [21], which took the membership, non-membership, and hesitancy into account simultaneously.

*Corresponding author: Han-Liang Huang, School of Mathematics and Statistics, Minnan Normal University, Zhangzhou 363000, P.R. China, e-mail: hl_huang1980.student@sina.com

Yuting Guo: Xiacang Middle School, Jizhou District, Tianjin, P.R. China 
The correlation coefficient formulas proposed by $\mathrm{Xu}[19,21]$ may have some limitations. First of all, the formulas [see Eqs. (1) and (2)] are expressed by the form of quotient, which is of 0/0 type if two IFSs are the same. Moreover, the results obtained by Xu's correlation coefficients are not coincident with our intuition in some situations. For example, the formulas may be equal to 1 even if $A_{1} \neq A_{2}$ (see example 1).

In this paper, we improve Xu's correlation coefficients so that it can overcome the drawbacks above. Two examples can show the effectivity of our proposed method. In Section 3, we introduce a new formula for calculating the correlation between IFSs and summarize the advantage of the improved correlation coefficient by comparing with Xu's correlation coefficients [19, 21]. We also generalize it to IVIFS environment. Furthermore, we show some applications of the improved correlation coefficient in medical diagnosis and clustering in Section 4.

\section{Preliminaries}

In this section, some basic concepts of the IFSs and IVIFSs are presented.

Definition 1 ([1]): Let $X$ be a universe of discourse, then an IFS $A$ is defined as:

$$
A=\left\{\left(x, \mu_{A}(x), v_{A}(x)\right) \mid x \in X\right\},
$$

where $\mu_{A}(x): X \rightarrow[0,1]$ and $v_{A}(x): X \rightarrow[0,1]$, with $0 \leq \mu_{A}(x)+v_{A}(x) \leq 1$. The numbers $\mu_{A}(x)$ and $v_{A}(x)$ represent the membership degree and the non-membership degree of the element $x$ to the set $A$, respectively. For each IFS $A$ over $X$, if $\pi_{A}(x)=1-\mu_{A}(x)-v_{A}(x), x \in X$, then $\pi_{A}(x)$ is called the hesitation degree of the element $x$ to the set $A$.

Let $\operatorname{IFS}(X)$ be the set of all IFSs over $X$. Then for $A_{1}, A_{2} \in \operatorname{IFS}(X)$, we have:

(1) $A_{1} \subseteq A_{2}$ if and only if $\mu_{A_{1}}(x) \leq \mu_{A_{2}}(x)$ and $v_{A_{1}}(x) \geq v_{A_{2}}(x)$ for each $x \in X$;

(2) $A_{1}=A_{2}$ if and only if $A_{1} \subseteq A_{2}$ and $A_{2} \subseteq A_{1}$.

Definition 2 ([2]): Let $X$ be a universe of discourse, then an IVIFS $\tilde{A}$ is defined as:

$$
\tilde{A}=\left\{\left(x, \tilde{\mu}_{\tilde{A}}(x), \tilde{v}_{\tilde{A}}(x)\right) \mid x \in X\right\}
$$

where $\tilde{\mu}_{\tilde{A}}(x)=\left[\tilde{\mu}_{\tilde{A}}^{L}(x), \tilde{\mu}_{\tilde{A}}^{U}(x)\right] \subseteq[0,1]$ and $\tilde{v}_{\tilde{A}}(x)=\left[\tilde{v}_{\tilde{A}}^{L}(x), \tilde{v}_{\tilde{A}}^{U}(x)\right] \subseteq[0,1]$ are intervals, and $0 \leq \tilde{\mu}_{\tilde{A}}^{U}(x)+\tilde{v}_{\tilde{A}}^{U}(x) \leq 1$ for each $x \in X$.

Especially, if $\tilde{\mu}_{\tilde{A}}^{L}(x)=\tilde{\mu}_{\tilde{A}}^{U}(x)$ and $\tilde{v}_{\tilde{A}}^{L}(x)=\tilde{v}_{\tilde{A}}^{U}(x)$, then the IVIFS $\tilde{A}$ is reduced to an ordinary IFS.

Let $\operatorname{IVIFS}(X)$ be the set of all IVIFSs over $X$. Then for $\tilde{A}_{1}, \tilde{A}_{2} \in \operatorname{IVIFS}(X)$, we have:

(1) $\tilde{A}_{1} \subseteq \tilde{A}_{2}$ if and only if $\tilde{\mu}_{\tilde{A}_{1}}^{U}(x) \leq \tilde{\mu}_{\tilde{A}_{2}}^{U}(x), \quad \tilde{\mu}_{\tilde{A}_{1}}^{L}(x) \leq \tilde{\mu}_{\tilde{A}_{2}}^{L}(x), \tilde{v}_{\tilde{A}_{1}}^{U}(x) \geq \tilde{v}_{\tilde{A}_{2}}^{U}(x)$ and $\tilde{v}_{\tilde{A}_{1}}^{L}(x) \geq \tilde{v}_{\tilde{A}_{2}}^{L}(x)$ for each $x \in X$;

(2) $\tilde{A}_{1}=\tilde{A}_{2}$ if and only if $\tilde{A}_{1} \subseteq \tilde{A}_{2}$ and $\tilde{A}_{2} \subseteq \tilde{A}_{1}$.

The correlation coefficient of two IFSs is introduced by $\mathrm{Xu}$ as follows.

Definition 3 ([19]): Let $A_{1}, A_{2} \in \operatorname{IFS}(X)$, and $X=\left\{x_{1}, x_{2}, \ldots, x_{n}\right\}$ be a finite universe of discourse, then we define

$$
\rho_{1}\left(A_{1}, A_{2}\right)=\frac{1}{2 n} \sum_{i=1}^{n}\left(\frac{\Delta u_{\text {min }}+\Delta u_{\text {max }}}{\Delta u_{i}+\Delta u_{\text {max }}}+\frac{\Delta v_{\text {min }}+\Delta v_{\text {max }}}{\Delta v_{i}+\Delta v_{\text {max }}}\right)
$$

as a correlation coefficient of the IFSs $A_{1}$ and $A_{2}$, where

$$
\begin{aligned}
& \Delta u_{i}=\left|\mu_{A_{1}}\left(x_{i}\right)-\mu_{A_{2}}\left(x_{i}\right)\right|, \quad \Delta v_{i}=\left|v_{A_{1}}\left(x_{i}\right)-v_{A_{2}}\left(x_{i}\right)\right| \quad(i=1,2, \cdots, n), \\
& \Delta u_{\min }=\min _{i}\left\{\left|\mu_{A_{1}}\left(x_{i}\right)-\mu_{A_{2}}\left(x_{i}\right)\right|\right\}, \Delta v_{\min }=\min _{i}\left\{\left|v_{A_{1}}\left(x_{i}\right)-v_{A_{2}}\left(x_{i}\right)\right|\right\}, \\
& \Delta u_{\max }=\max _{i}\left\{\left|\mu_{A_{1}}\left(x_{i}\right)-\mu_{A_{2}}\left(x_{i}\right)\right|\right\}, \Delta v_{\max }=\max _{i}\left\{\left|v_{A_{1}}\left(x_{i}\right)-v_{A_{2}}\left(x_{i}\right)\right|\right\} .
\end{aligned}
$$


IFS contains three elements, such as membership, non-membership, and hesitation. However, the above correlation coefficient of Definition 3 does not take the hesitation into account. Thus, Xu improved it in Ref. [21]:

$$
\rho_{2}\left(A_{1}, A_{2}\right)=\frac{1}{3 n} \sum_{i=1}^{n}\left(\frac{\Delta u_{\text {min }}+\Delta u_{\text {max }}}{\Delta u_{i}+\Delta u_{\text {max }}}+\frac{\Delta v_{\text {min }}+\Delta v_{\text {max }}}{\Delta v_{i}+\Delta v_{\text {max }}}+\frac{\Delta \pi_{\text {min }}+\Delta \pi_{\max }}{\Delta \pi_{i}+\Delta \pi_{\max }}\right),
$$

where

$$
\begin{aligned}
& \pi_{A_{1}}\left(x_{i}\right)=1-\mu_{A_{1}}\left(x_{i}\right)-v_{A_{1}}\left(x_{i}\right), \pi_{A_{2}}\left(x_{i}\right)=1-\mu_{A_{2}}\left(x_{i}\right)-v_{A_{2}}\left(x_{i}\right), \\
& \Delta \pi_{i}=\left|\pi_{A_{1}}\left(x_{i}\right)-\pi_{A_{2}}\left(x_{i}\right)\right|, \Delta \pi_{\min }=\min _{i}\left\{\left|\pi_{A_{1}}\left(x_{i}\right)-\pi_{A_{2}}\left(x_{i}\right)\right|\right\}, \\
& \Delta \pi_{\max }=\max _{i}\left\{\left|\pi_{A_{1}}\left(x_{i}\right)-\pi_{A_{2}}\left(x_{i}\right)\right|\right\} \quad(i=1,2, \cdots, n) .
\end{aligned}
$$

Remark 1: The above correlation coefficients $\rho_{k}\left(A_{i}, A_{j}\right) \quad(k=1,2)$ satisfy the following properties:

(a) $0 \leq \rho_{k}\left(A_{i}, A_{j}\right) \leq 1$;

(b) $\rho_{k}\left(A_{i}, A_{j}\right)=\rho_{k}\left(A_{j}, A_{i}\right) \quad(i, j=1,2, \ldots, m)$.

Clustering analysis is an important application of correlation coefficient. In what follows, we recall some concepts related to clustering analysis such as association matrix and equivalent association matrix.

Definition 4 ([22]): Let $A_{j} \in \operatorname{IFS}(X),(j=1,2, \ldots, m)$, then $C=\left(c_{i j}\right)_{m \times m}$ is called an association matrix, where $c_{i j}=\rho\left(A_{i}, A_{j}\right) \quad(i, j=1,2, \ldots, m)$ is the correlation coefficient of $A_{i}$ and $A_{j}$, which have the following properties:

(a) $0 \leq c_{i j} \leq 1 \quad(i, j=1,2, \ldots, m)$;

(b) $c_{i j}=c_{j i}(i, j=1,2, \ldots m)$;

(c) $c_{i j}=1$ if and only if $A_{i}=A_{j}$.

Definition 5 ([22]): Let $C=\left(c_{i j}\right)_{m \times m}$ be an association matrix, if

$$
C^{2}=C \circ C=\left(\bar{C}_{i j}\right)_{m \times m},
$$

then $C^{2}$ is called a composition matrix of $C$, where $\bar{c}_{i j}=\max _{k}\left\{\min \left\{c_{i k}, c_{k j}\right\}\right\} \quad(i, j=1,2, \ldots, m)$.

Definition 6 ([22]): Let $C=\left(c_{i j}\right)_{m \times m}$ be an association matrix, and $C^{2}$ be its composition matrix, if $C^{2} \subseteq C$, i.e.,

$$
\max _{k}\left\{\min \left\{c_{i k}, c_{k j}\right\}\right\} \leq c_{i j}
$$

for all $i, j=1,2, \ldots, m$, then $C$ is called an equivalent association matrix.

By the transitivity principle of equivalent matrix [17], it is easy to prove the following:

Theorem 1 ([22]): Let $C=\left(c_{i j}\right)_{m \times m}$ be an association matrix, then after the finite times of compositions:

$$
C \rightarrow C^{2} \rightarrow C^{4} \rightarrow \cdots C^{2^{k}} \rightarrow \cdots
$$

there must exist a positive integer $k$ such that $C^{2^{k}}=C^{2^{(k+1)}}$, and $C^{2^{k}}$ is an equivalent association matrix.

Definition 7 ([22]): Let $C=\left(c_{i j}\right)_{m \times m}$ be an equivalent association matrix, then we call $C_{\lambda}=\left({ }_{\lambda} c_{i j}\right)_{m \times m}$ the $\lambda$-cutting matrix of $C$, where 


$$
{ }_{\lambda} c_{i j}=\left\{\begin{array}{l}
0, c_{i j}<\lambda ; \\
1, c_{i j} \geq \lambda .
\end{array} \quad(i, j=1,2, \cdots, m)\right.
$$

and $\lambda$ is the confidence level with $\lambda \in[0,1]$.

\section{Main Results}

In this section, we will introduce an improved correlation coefficient and generalize it to IVIFS environment.

The correlation coefficients proposed by $\mathrm{Xu}[20,21]$ share the two properties of Remark 1 . However, both the correlation coefficient formulas have the following limitations:

1. The quotients in formulas (1) and (2) are of the $0 / 0$ type if the formulas satisfy $A_{1}=A_{2}$, and it is unmeaningful in mathematical logic. Although we can deliberately set a special value for this $0 / 0$ type quotient, it is more reasonable to avoid emerging this case.

2. Formula (1) as well as the improved formula (2) may be equal to 1 even if $A_{1} \neq A_{2}$. Hence, neither Formula (1) nor (2) satisfies the condition: $\rho\left(A_{i}, A_{j}\right)=1 \Leftrightarrow A_{i}=A_{i}$, then the two formulas cannot be applied in clustering sometimes. Let us employ an example to illustrate our idea.

Example 1: Let $A_{1}$ and $A_{2}$ be two IFSs in $X=\left\{x_{1}, x_{2}\right\}$ given by

$$
\begin{aligned}
& A_{1}=\left\{\left\langle x_{1}, 0.4,0.3\right\rangle,\left\langle x_{2}, 0.3,0.2\right\rangle\right\} ; \\
& A_{2}=\left\{\left\langle x_{1}, 0.3,0.2\right\rangle,\left\langle x_{2}, 0.2,0.1\right\rangle\right\} .
\end{aligned}
$$

Obviously, $A_{1} \neq A_{2}$. However, $\rho_{1}\left(A_{1}, A_{2}\right)=1$ and $\rho_{2}\left(A_{1}, A_{2}\right)=1$.

Thus, how to derive the correlation coefficients of IFSs satisfying the desirable property above $\left(\rho\left(A_{i}\right.\right.$, $\left.A_{j}\right)=1 \Leftrightarrow A_{i}=A_{j}$ ) is an interesting research topic. In order to solve this problem, we develop a new definition of correlation coefficient of the IFSs.

\subsection{An Improved Correlation Coefficient of the IFSs}

In the following, we define an improved correlation coefficient of the IFSs.

Definition 8: Let $A_{1}, A_{2} \in \operatorname{IFS}(X)$, and $X=\left\{x_{1}, x_{2}, \ldots, x_{n}\right\}$ be a finite universe of discourse, then we define

$$
\rho^{*}\left(A_{1}, A_{2}\right)=\frac{1}{2 n} \sum_{i=1}^{n}\left[\alpha_{i}\left(1-\Delta \mu_{i}\right)+\beta_{i}\left(1-\Delta v_{i}\right)\right]
$$

as a correlation coefficient of the IFSs $A_{1}$ and $A_{2}$, where

$$
\begin{aligned}
& \alpha_{i}=\frac{c-\Delta u_{i}-\Delta u_{\text {max }}}{c-\Delta \mu_{\min }-\Delta u_{\text {max }}}, \beta_{i}=\frac{c-\Delta v_{i}-\Delta v_{\text {max }}}{c-\Delta v_{\text {min }}-\Delta v_{\text {max }}} \quad(c>2, i=1,2, \cdots, n), \\
& \Delta u_{i}=\left|\mu_{A_{1}}\left(x_{i}\right)-\mu_{A_{2}}\left(x_{i}\right)\right|, \quad \Delta v_{i}=\left|v_{A_{1}}\left(x_{i}\right)-v_{A_{2}}\left(x_{i}\right)\right|, \\
& \Delta u_{\text {min }}=\min _{i}\left\{\left|\mu_{A_{1}}\left(x_{i}\right)-\mu_{A_{2}}\left(x_{i}\right)\right|\right\}, \quad \Delta v_{\text {min }}=\min _{i}\left\{\left|v_{A_{1}}\left(x_{i}\right)-v_{A_{2}}\left(x_{i}\right)\right|\right\}, \\
& \Delta u_{\text {max }}=\max _{i}\left\{\left|\mu_{A_{1}}\left(x_{i}\right)-\mu_{A_{2}}\left(x_{i}\right)\right|\right\}, \Delta v_{\text {max }}=\max _{i}\left\{\left|v_{A_{1}}\left(x_{i}\right)-v_{A_{2}}\left(x_{i}\right)\right|\right\} .
\end{aligned}
$$

Remark 2: Note that the condition " $c>2$ " ensures that both $\alpha_{i}$ and $\beta_{i}$ belong to $(0,1)$. Hence, the correlation coefficient $\rho^{*}\left(A_{1}, A_{2}\right)$ satisfies the condition $0 \leq \rho^{*}\left(A_{1}, A_{2}\right) \leq 1$. If some $\alpha_{i}$ and $\beta_{i}$ are $<0$, it will cause $\rho^{*}\left(A_{1}, A_{2}\right)<0$. We can see example 2 as follows. 
Example 2: Let $A_{1}$ and $A_{2}$ be two IFSs in $X=\left\{x_{1}, x_{2}\right\}$ given by

$$
\begin{aligned}
& A_{1}=\left\{\left\langle x_{1}, 0.9,0.1\right\rangle,\left\langle x_{2}, 0.7,0.2\right\rangle\right\} \\
& A_{2}=\left\{\left\langle x_{1}, 0.1,0.8\right\rangle,\left\langle x_{2}, 0.6,0.4\right\rangle\right\} .
\end{aligned}
$$

By Eq. (3), we can have $\alpha_{1}=-6, \alpha_{2}=1, \beta_{1}=-4, \beta_{2}=1$ when $c=1$, then we can calculate that $\rho_{1}\left(A_{1}, A_{2}\right)$ $=-0.175<0$.

We present some properties of the improved correlation coefficient in the following.

Theorem 2: The correlation coefficient $\rho^{\star}\left(A_{1}, A_{2}\right)$ satisfies the following properties:

(a) $\rho^{\star}\left(A_{1}, A_{2}\right)=\rho^{*}\left(A_{2}, A_{1}\right)$;

(b) $0 \leq \rho^{\star}\left(A_{1}, A_{2}\right) \leq 1$;

(c) $A_{1}=A_{2} \Leftrightarrow \rho^{*}\left(A_{1}, A_{2}\right)=1$.

Proof

(a) Obviously.

(b) Since $0<\alpha_{i} \leq 1,0<\beta_{i} \leq 1$ and $0 \leq 1-\Delta \mu_{i} \leq 1,0 \leq 1-\Delta v_{i} \leq 1$, then

$$
0 \leq \alpha_{i}\left(1-\Delta \mu_{i}\right)+\beta_{i}\left(1-\Delta v_{i}\right) \leq 2 \quad(i=1,2, \cdots, n),
$$

and thus, by Eq. (3), we know that $0 \leq \rho^{\star}\left(A_{1}, A_{2}\right) \leq 1$.

(c) " $\Rightarrow$ " If $A_{1}=A_{2}$, it implies that: $\mu_{A_{1}}\left(x_{i}\right)=\mu_{A_{2}}\left(x_{i}\right), v_{A_{1}}\left(x_{i}\right)=v_{A_{2}}\left(x_{i}\right)$ for all $x_{i} \in X(i=1,2, \ldots, n)$, then

$$
\Delta u_{i}=\Delta u_{\min }=\Delta u_{\max }=0, \Delta v_{i}=\Delta v_{\min }=\Delta v_{\max }=0 \text {. }
$$

Thus, $\rho^{\star}\left(A_{1}, A_{2}\right)=1$.

" $\Leftarrow$ " If $\rho^{*}\left(A_{1}, A_{2}\right)=1$, by the fact that

$$
0 \leq \alpha_{i}\left(1-\Delta \mu_{i}\right) \leq 1, \quad 0 \leq \beta_{i}\left(1-\Delta v_{i}\right) \leq 1,
$$

we have

$$
\alpha_{i}\left(1-\Delta \mu_{i}\right)+\beta_{i}\left(1-\Delta v_{i}\right)=2 \text { and } \alpha_{i}\left(1-\Delta \mu_{i}\right)=\beta_{i}\left(1-\Delta v_{i}\right)=1 \quad(i=1,2, \cdots, n) .
$$

Since

$$
0<\alpha_{i} \leq 1, \quad 0<\beta_{i} \leq 1, \quad 0 \leq 1-\Delta u_{i} \leq 1, \quad 0 \leq 1-\Delta v_{i} \leq 1,
$$

then we obtain

$$
\alpha_{i}=\beta_{i}=1,1-\Delta u_{i}=1,1-\Delta v_{i}=1 \text {, i.e., } \Delta u_{i}=\Delta v_{i}=0 \text {. }
$$

Thus, $\mu_{A_{1}}\left(x_{i}\right)=\mu_{A_{2}}\left(x_{i}\right), v_{A_{1}}\left(x_{i}\right)=v_{A_{2}}\left(x_{i}\right)$ for all $x_{i} \in X$, i.e., $A_{1}=A_{2}$.

Remark 3: We may draw the conclusion from Theorem 2 that the improved correlation coefficient overcomes the limitations mentioned above of Xu's correlation coefficients.

First of all, the improved correlation coefficient avoids emerging the 0/0 type quotient, which is more reasonable in mathematical logic.

In addition, under the condition $A_{1} \neq A_{2}$, the improved correlation coefficient $\rho^{\star}\left(A_{1}, A_{2}\right)$ is certainly less than one by the third property of Theorem 3, which is coincident with our intuition. For instance, in Example 1 , $A_{1} \neq A_{2}$ and $\rho^{\star}\left(A_{1}, A_{2}\right)=0.9<1$. Furthermore, the improved correlation coefficient can guarantee that the correlation coefficient of any two IFSs equals 1 if and only if these two IFSs are the same. Consequently, it can be applied in medical diagnosis and clustering effectively, which will be discussed in Section 4.

In many situations, the weight of every element $x_{i} \in X$ should be taken into account. For example, in the multiple attribute decision-making problems, each attribute usually has different importance and, thus, needs to be assigned a different weight. As a result, we further extend Formula (3). 
Definition 9: Let $A_{1}, A_{2} \in \operatorname{IFS}(X)$, and $X=\left\{x_{1}, x_{2}, \ldots, x_{n}\right\}$ be a finite universe of discourse, then we define

$$
\rho_{1}^{*}\left(A_{1}, A_{2}\right)=\frac{1}{2} \sum_{i=1}^{n}\left\{\omega_{i}\left[\alpha_{i}\left(1-\Delta \mu_{i}\right)+\beta_{i}\left(1-\Delta v_{i}\right)\right]\right\}
$$

as a correlation coefficient of the IFSs $A_{1}$ and $A_{2}$, where

$$
\alpha_{i}=\frac{c-\Delta \mu_{i}-\Delta u_{\max }}{c-\Delta \mu_{\min }-\Delta \mu_{\max }}, \beta_{i}=\frac{c-\Delta v_{i}-\Delta v_{\max }}{c-\Delta v_{\min }-\Delta v_{\max }} \quad(c>2),
$$

the weight vector of $x_{i}(i=1,2, \ldots, n)$ is $\omega=\left(\omega_{1}, \omega_{2}, \ldots, \omega_{n}\right)^{\mathrm{T}}, \omega_{i} \geq 0 \quad(i=1,2, \ldots, n)$ and $\sum_{i=1}^{n} \omega_{i}=1$. The value of $\omega_{i}$ can be determined by several methods, such as statistical distribution, analytic hierarchy process, coefficient of variation method, and so on. It can also be determined according to the experts' opinions. Especially, if $\omega=\left(\frac{1}{n}, \frac{1}{n}, \cdots, \frac{1}{n}\right)^{\mathrm{T}}$, Formula (4) reduces to Formula (3).

\subsection{The Improved Correlation Coefficient of the IVIFSs}

In the following, we generalize the idea of Definition 8 to the IVIFS theory.

Definition 10: Let $\tilde{A}_{1}, \tilde{A}_{2} \in \operatorname{IVIFS}(X)$, and $X=\left\{x_{1}, x_{2}, \ldots, x_{n}\right\}$ be a finite universe of discourse, then we define

$$
\rho_{2}^{*}\left(\tilde{A}_{1}, \tilde{A}_{2}\right)=\frac{1}{4 n} \sum_{i=1}^{n}\left[\gamma_{i}\left(1-\Delta \tilde{\mu}_{i}^{L}\right)+\zeta_{i}\left(1-\Delta \tilde{u}_{i}^{U}\right)+\varphi_{i}\left(1-\Delta \tilde{v}_{i}^{L}\right)+\psi_{i}\left(1-\Delta \tilde{v}_{i}^{U}\right)\right]
$$

as a correlation coefficient of IVIFSs $\tilde{A}_{1}$ and $\tilde{A}_{2}$, where

$$
\begin{aligned}
& \gamma_{i}=\frac{c-\Delta \tilde{\mu}_{i}^{L}-\Delta \tilde{\mu}_{\max }^{L}}{c-\Delta \tilde{\mu}_{\min }^{L}-\Delta \tilde{\mu}_{\max }^{L}}, \quad \zeta_{i}=\frac{c-\Delta \tilde{\mu}_{i}^{U}-\Delta \tilde{\mu}_{\max }^{U}}{c-\Delta \tilde{\mu}_{\min }^{U}-\Delta \tilde{\mu}_{\max }^{U},} \\
& \varphi_{i}=\frac{c-\Delta \tilde{v}_{i}^{L}-\Delta \tilde{v}_{\max }^{L}}{c-\Delta \tilde{v}_{\min }^{L}-\Delta \tilde{v}_{\max }^{L}}, \quad \psi_{i}=\frac{c-\Delta \tilde{v}_{i}^{U}-\Delta \tilde{v}_{\max }^{U}}{c-\Delta \tilde{v}_{\min }^{U}-\Delta \tilde{v}_{\max }^{U}}(c>2), \\
& \Delta \tilde{\mu}_{i}^{L}=\left|\tilde{\mu}_{\tilde{A}_{1}}^{L}\left(x_{i}\right)-\tilde{\mu}_{\tilde{A}_{2}}^{L}\left(x_{i}\right)\right|, \Delta \tilde{v}_{i}^{L}=\left|\tilde{v}_{\tilde{A}_{1}}^{L}\left(x_{i}\right)-\tilde{v}_{\tilde{A}_{2}}^{L}\left(x_{i}\right)\right|, \\
& \Delta \tilde{\mu}_{i}^{U}=\left|\tilde{\mu}_{\tilde{A}_{1}}^{U}\left(x_{i}\right)-\tilde{\mu}_{\tilde{A}_{2}}^{U}\left(x_{i}\right)\right|, \Delta \tilde{v}_{i}^{U}=\left|\tilde{v}_{\tilde{A}_{1}}^{U}\left(x_{i}\right)-\tilde{v}_{\tilde{A}_{2}}^{U}\left(x_{i}\right)\right|(i=1,2, \cdots, n), \\
& \Delta \tilde{\mu}_{\min }^{L}=\min _{i}\left\{\left|\tilde{\mu}_{\tilde{A}_{1}}^{L}\left(x_{i}\right)-\tilde{\mu}_{\tilde{A}_{2}}^{L}\left(x_{i}\right)\right|\right\}, \Delta \tilde{\mu}_{\min }^{U}=\min _{i}\left\{\left|\tilde{\mu}_{\tilde{A}_{1}}^{U}\left(x_{i}\right)-\tilde{\mu}_{\tilde{A}_{2}}^{U}\left(x_{i}\right)\right|\right\}, \\
& \Delta \tilde{\mu}_{\max }^{L}=\max _{i}\left\{\left|\tilde{\mu}_{\tilde{A}_{1}}^{L}\left(x_{i}\right)-\tilde{\mu}_{\tilde{A}_{2}}^{L}\left(x_{i}\right)\right|\right\}, \Delta \tilde{\mu}_{\max }^{U}=\max _{i}\left\{\left|\tilde{\mu}_{\tilde{A}_{1}}^{U}\left(x_{i}\right)-\tilde{\mu}_{\tilde{A}_{2}}^{U}\left(x_{i}\right)\right|\right\}, \\
& \Delta \tilde{v}_{\min }^{L}=\min _{i}\left\{\left|\tilde{v}_{\tilde{A}_{1}}^{L}\left(x_{i}\right)-\tilde{v}_{\tilde{A}_{2}}^{L}\left(x_{i}\right)\right|\right\}, \Delta \tilde{v}_{\min }^{U}=\min _{i}\left\{\left|\tilde{v}_{\tilde{A}_{1}}^{U}\left(x_{i}\right)-\tilde{v}_{\tilde{A}_{2}}^{U}\left(x_{i}\right)\right|\right\}, \\
& \Delta \tilde{v}_{\max }^{L}=\max _{i}\left\{\left|\tilde{v}_{\tilde{A}_{1}}^{L}\left(x_{i}\right)-\tilde{v}_{\tilde{A}_{2}}^{L}\left(x_{i}\right)\right|\right\}, \Delta \tilde{v}_{\max }^{U}=\max _{i}\left\{\left|\tilde{v}_{\tilde{A}_{1}}^{U}\left(x_{i}\right)-\tilde{v}_{\tilde{A}_{2}}^{U}\left(x_{i}\right)\right|\right\} .
\end{aligned}
$$

Equation (5) also can be generalized to more general cases:

$$
\rho_{3}^{*}\left(\tilde{A}_{1}, \tilde{A}_{2}\right)=\frac{1}{4} \sum_{i=1}^{n}\left\{\omega_{i}\left[\gamma_{i}\left(1-\Delta \tilde{\mu}_{i}^{L}\right)+\zeta_{i}\left(1-\Delta \tilde{\mu}_{i}^{U}\right)+\varphi_{i}\left(1-\Delta \tilde{v}_{i}^{L}\right)+\psi_{i}\left(1-\Delta \tilde{v}_{i}^{U}\right)\right]\right\},
$$

where $\omega=\left(\omega_{1}, \omega_{2}, \ldots, \omega_{n}\right)^{\mathrm{T}}, \omega_{i} \geq 0(i=1,2, \ldots, n)$ is the weight of $x_{i}(i=1,2, \ldots, n)$ and $\sum_{i=1}^{n} \omega_{i}=1$. The value of $\omega_{i}$ can be determined by the same way which was mentioned in Definition 9. Especially, if $\omega=\left(\frac{1}{n}, \frac{1}{n}, \cdots, \frac{1}{n}\right)^{\mathrm{T}}$, Formula (6) reduces to Formula (5). 
Theorem 3: The correlation coefficient $\rho_{2}^{*}\left(\tilde{A}_{1}, \tilde{A}_{2}\right)$ satisfies the following properties:

(a) $\rho_{2}^{*}\left(\tilde{A}_{1}, \tilde{A}_{2}\right)=\rho_{2}^{*}\left(\tilde{A}_{2}, \tilde{A}_{1}\right)$;

(b) $0 \leq \rho_{2}^{*}\left(\tilde{A}_{1}, \tilde{A}_{2}\right) \leq 1$;

(c) $\tilde{A}_{1}=\tilde{A}_{2} \Leftrightarrow \rho_{2}^{*}\left(\tilde{A}_{1}, \tilde{A}_{2}\right)=1$.

\section{Proof}

(a) Obviously.

(b) Since

$$
0<\gamma_{i}<1,0<\zeta \leq 1,0<\varphi_{i} \leq 1,0<\psi_{i} \leq 1
$$

and

$$
0 \leq 1-\Delta \tilde{\mu}_{i}^{L} \leq 1,0 \leq 1-\Delta \tilde{\mu}_{i}^{U} \leq 1,0 \leq 1-\Delta \tilde{v}_{i}^{L} \leq 1,0 \leq 1-\Delta \tilde{v}_{i}^{U} \leq 1,
$$

then

$$
0 \leq \gamma_{i}\left(1-\Delta \tilde{u}_{i}^{L}\right)+\zeta_{i}\left(1-\Delta \tilde{\mu}_{i}^{U}\right)+\varphi_{i}\left(1-\Delta \tilde{v}_{i}^{L}\right)+\psi_{i}\left(1-\Delta \tilde{v}_{i}^{U}\right) \leq 4
$$

where $i=1,2, \ldots, n$. Thus, by Eq. (5), we know that $0 \leq \rho_{2}^{*}\left(\tilde{A}_{1}, \tilde{A}_{2}\right) \leq 1$.

(c) “ $\Rightarrow$ ” If $\tilde{A}_{1}=\tilde{A}_{2}$, it implies that: $\tilde{\mu}_{\tilde{A}_{1}}^{L}\left(x_{i}\right)=\tilde{\mu}_{\tilde{A}_{2}}^{L}\left(x_{i}\right), \tilde{\mu}_{\tilde{A}_{1}}^{U}\left(x_{i}\right)=\tilde{\mu}_{\tilde{A}_{2}}^{U}\left(x_{i}\right), \tilde{v}_{\tilde{A}_{1}}^{L}\left(x_{i}\right)=\tilde{v}_{\tilde{A}_{2}}^{L}\left(x_{i}\right), \tilde{v}_{\tilde{A}_{1}}^{U}\left(x_{i}\right)=\tilde{v}_{\tilde{A}_{2}}^{U}\left(x_{i}\right)$ for all $x_{i} \in X$, then

$$
\begin{aligned}
& \Delta \tilde{u}_{i}^{L}=\Delta \tilde{\mu}_{\text {min }}^{L}=\Delta \tilde{\mu}_{\text {max }}^{L}=0, \quad \Delta \tilde{\mu}_{i}^{U}=\Delta \tilde{\mu}_{\text {min }}^{U}=\Delta \tilde{\mu}_{\text {max }}^{U}=0, \\
& \Delta \tilde{v}_{i}^{L}=\Delta \tilde{v}_{\text {min }}^{L}=\Delta \tilde{v}_{\text {max }}^{L}=0, \Delta \tilde{v}_{i}^{U}=\Delta \tilde{v}_{\text {min }}^{U}=\Delta \tilde{v}_{\text {max }}^{U}=0 .
\end{aligned}
$$

Thus, $\rho_{2}^{*}\left(\tilde{A}_{1}, \tilde{A}_{2}\right)=1$.

" $\Leftarrow$ " If $\rho_{2}^{*}\left(\tilde{A}_{1}, \tilde{A}_{2}\right)=1$, by the fact that

$$
\begin{aligned}
& 0 \leq \gamma_{i}\left(1-\Delta \tilde{\mu}_{i}^{L}\right) \leq 1,0 \leq \zeta_{i}\left(1-\Delta \tilde{\mu}_{i}^{U}\right) \leq 1, \\
& 0 \leq \varphi_{i}\left(1-\Delta \tilde{v}_{i}^{L}\right) \leq 1,0 \leq \psi_{i}\left(1-\Delta \tilde{v}_{i}^{U}\right) \leq 1,
\end{aligned}
$$

we have

$$
\gamma_{i}\left(1-\Delta \tilde{\mu}_{i}^{L}\right)+\zeta_{i}\left(1-\Delta \tilde{\mu}_{i}^{U}\right)+\varphi_{i}\left(1-\Delta \tilde{v}_{i}^{L}\right)+\psi_{i}\left(1-\Delta \tilde{v}_{i}^{U}\right)=4
$$

and

$$
\gamma_{i}\left(1-\Delta \tilde{\mu}_{i}^{L}\right)=\zeta_{i}\left(1-\Delta \tilde{\mu}_{i}^{U}\right)=\varphi_{i}\left(1-\Delta \tilde{v}_{i}^{L}\right)=\psi_{i}\left(1-\Delta \tilde{v}_{i}^{U}\right)=1 .
$$

Since

$$
0<\gamma_{i}<1,0<\zeta \leq 1,0<\varphi_{i} \leq 1,0<\psi_{i} \leq 1
$$

and

$$
0 \leq 1-\Delta \tilde{\mu}_{i}^{L} \leq 1, \quad 0 \leq 1-\Delta \tilde{\mu}_{i}^{U} \leq 1, \quad 0 \leq 1-\Delta \tilde{v}_{i}^{L} \leq 1, \quad 0 \leq 1-\Delta \tilde{v}_{i}^{U} \leq 1,
$$

then, we obtain

$$
1-\Delta \tilde{\mu}_{i}^{L}=1-\Delta \tilde{\mu}_{i}^{U}=1-\Delta \tilde{v}_{i}^{L}=1-\Delta \tilde{v}_{i}^{U}=1,
$$

i.e.,

$$
\Delta \tilde{\mu}_{i}^{L}=\Delta \tilde{\mu}_{i}^{U}=\Delta \tilde{v}_{i}^{L}=\Delta \tilde{v}_{i}^{U}=0 .
$$

Thus, 


$$
\begin{aligned}
& \tilde{\mu}_{\tilde{A}_{1}}^{L}\left(x_{i}\right)=\tilde{\mu}_{\tilde{A}_{2}}^{L}\left(x_{i}\right), \quad \tilde{\mu}_{\tilde{A}_{1}}^{U}\left(x_{i}\right)=\tilde{\mu}_{\tilde{A}_{2}}^{U}\left(x_{i}\right), \\
& \tilde{v}_{\tilde{A}_{1}}^{L}\left(x_{i}\right)=\tilde{v}_{\tilde{A}_{2}}^{L}\left(x_{i}\right), \quad \tilde{v}_{\tilde{A}_{1}}^{U}\left(x_{i}\right)=\tilde{v}_{\tilde{A}_{2}}^{U}\left(x_{i}\right),
\end{aligned}
$$

for all $x_{i} \in X$, i.e., $\tilde{A}_{1}=\tilde{A}_{2}$.

\section{Applications of the Improved Correlation Coefficient}

In this section, we mainly focus on the applications of the improved correlation coefficient in medical diagnosis and clustering.

\subsection{The Application in Medical Diagnosis}

First, for the set of diagnosis $A=\left\{A_{1}, A_{2}, \ldots, A_{n}\right\}$, the set of symptoms $C=\left\{C_{1}, C_{2}, \ldots, C_{t}\right\}$, and the set of patients $B=\left\{B_{1}, B_{2}, \ldots, B_{m}\right\}$, suppose the weight of $C_{k}$ is $\omega_{k} \quad(k=1,2, \ldots, t)$, then the diagnosis steps are as follows:

- Step 1: Based on the experience of experts, each symptom is described by a pair of parameters $(\mu, v)$, i.e., the membership $\mu$ and the non-membership $v$.

- Step 2: For every patient $B_{j} \quad(j=1,2, \ldots, m)$, according to Formula (4), we can calculate the correlation coefficient $\rho_{1}^{*}\left(A_{i}, B_{j}\right),(i=1,2, \cdots, n)$, where $A_{i} \quad(i=1,2, \ldots, n)$ are the diagnosis results.

- Step 3: For every patient $B_{j} \quad(j=1,2, \ldots, m)$, select the diagnosis result $A_{i_{0}}$ in $A$ most close to the patient $B_{j}$, i.e., $\rho_{1}^{*}\left(A_{i 0}, B_{j}\right)=\max \left\{\rho_{1}^{*}\left(A_{i}, B_{j}\right) \mid A_{i} \in A\right\}$. Hence, it can be asserted that the diagnosis result of patient $B_{j}$ is $A_{i_{0}}$.

Example 3 ([14]): To make a proper diagnosis $A$ for a patient with the given values of symptoms $C$, a medical knowledge base is necessary that involves elements described in terms of IFSs. The set of symptoms is

$$
C=\left\{C_{1}, C_{2}, C_{3}, C_{4}, C_{5}\right\}=\{\text { Temperature, Headache, Stomach pain, Cough, Chest pain }\},
$$

the set of diagnosis is

$$
A=\left\{A_{1}, A_{2}, A_{3}, A_{4}, A_{5}\right\}=\{\text { Viral fever, Malaria, Typhoid, Stomach problem, Chest problem }\}
$$

and the set of patients is

$$
B=\left\{B_{1}, B_{2}, B_{3}, B_{4}\right\}=\{A l, \text { Bob, Joe }, T e d\}
$$

$\omega=\left(\omega_{1}, \omega_{2}, \ldots, \omega_{5}\right)^{\mathrm{T}}$ is the weight vector of symptoms, let $\omega_{1}=\omega_{2}=\cdots=\omega_{5}=\frac{1}{5}$. The data are given in Table 1 - each symptom is described by a pair of parameters $(\mu, v)$, i.e., the membership $\mu$ and the non-membership $v$. The symptoms are given in Table 2 . We need to seek a diagnosis for each patient $B_{j}(j=1,2,3,4)$.

Table 1: Symptoms Characteristic for the Considered Diagnoses.

\begin{tabular}{rrrrrr}
\hline & $\boldsymbol{A}_{1}$ & $\boldsymbol{A}_{2}$ & $\boldsymbol{A}_{3}$ & $\boldsymbol{A}_{4}$ & $\boldsymbol{A}_{5}$ \\
\hline$C_{1}$ & $(0.4,0.0)$ & $(0.7,0.0)$ & $(0.3,0.3)$ & $(0.1,0.7)$ & $(0.1,0.8)$ \\
$C_{2}$ & $(0.3,0.5)$ & $(0.2,0.6)$ & $(0.6,0.1)$ & $(0.2,0.4)$ & $(0.0,0.8)$ \\
$C_{3}$ & $(0.1,0.7)$ & $(0.0,0.9)$ & $(0.2,0.7)$ & $(0.8,0.0)$ & $(0.2,0.8)$ \\
$C_{4}$ & $(0.4,0.3)$ & $(0.7,0.0)$ & $(0.2,0.6)$ & $(0.2,0.7)$ & $(0.2,0.8)$ \\
$C_{5}$ & $(0.1,0.7)$ & $(0.1,0.8)$ & $(0.1,0.9)$ & $(0.2,0.7)$ & $(0.8,0.1)$ \\
\hline
\end{tabular}


Table 2: Symptoms Characteristic for the Considered Patients.

\begin{tabular}{rrrrrr}
\hline & $C_{1}$ & $C_{2}$ & $C_{3}$ & $C_{4}$ & $C_{5}$ \\
\hline$B_{1}$ & $(0.8,0.1)$ & $(0.6,0.1)$ & $(0.2,0.8)$ & $(0.6,0.1)$ & $(0.1,0.6)$ \\
$B_{2}$ & $(0.0,0.8)$ & $(0.4,0.4)$ & $(0.6,0.1)$ & $(0.1,0.7)$ & $(0.1,0.8)$ \\
$B_{3}$ & $(0.8,0.1)$ & $(0.8,0.1)$ & $(0.0,0.6)$ & $(0.2,0.7)$ & $(0.0,0.5)$ \\
$B_{4}$ & $(0.6,0.1)$ & $(0.5,0.4)$ & $(0.3,0.4)$ & $(0.7,0.2)$ & $(0.3,0.4)$ \\
\hline
\end{tabular}

First, construct IFSs:

$$
\begin{aligned}
& A_{1}=\left\{\left\langle C_{1}, 0.4,0.0\right\rangle,\left\langle C_{2}, 0.3,0.5\right\rangle,\left\langle C_{3}, 0.1,0.7\right\rangle,\left\langle C_{4}, 0.4,0.3\right\rangle,\left\langle C_{5}, 0.1,0.7\right\rangle\right\}, \\
& A_{2}=\left\{\left\langle C_{1}, 0.7,0.0\right\rangle,\left\langle C_{2}, 0.2,0.6\right\rangle,\left\langle C_{3}, 0.0,0.9\right\rangle,\left\langle C_{4}, 0.7,0.0\right\rangle,\left\langle C_{5}, 0.1,0.8\right\rangle\right\}, \\
& A_{3}=\left\{\left\langle C_{1}, 0.3,0.3\right\rangle,\left\langle C_{2}, 0.6,0.1\right\rangle,\left\langle C_{3}, 0.2,0.7\right\rangle,\left\langle C_{4}, 0.2,0.6\right\rangle,\left\langle C_{5}, 0.1,0.9\right\rangle\right\}, \\
& A_{4}=\left\{\left\langle C_{1}, 0.1,0.7\right\rangle,\left\langle C_{2}, 0.2,0.4\right\rangle,\left\langle C_{3}, 0.8,0.0\right\rangle,\left\langle C_{4}, 0.2,0.7\right\rangle,\left\langle C_{5}, 0.2,0.7\right\rangle\right\}, \\
& A_{5}=\left\{\left\langle C_{1}, 0.1,0.8\right\rangle,\left\langle C_{2}, 0.0,0.8\right\rangle,\left\langle C_{3}, 0.2,0.8\right\rangle,\left\langle C_{4}, 0.2,0.8\right\rangle,\left\langle C_{5}, 0.8,0.1\right\rangle\right\}, \\
& B_{1}=\left\{\left\langle C_{1}, 0.8,0.1\right\rangle,\left\langle C_{2}, 0.6,0.1\right\rangle,\left\langle C_{3}, 0.2,0.8\right\rangle,\left\langle C_{4}, 0.6,0.1\right\rangle,\left\langle C_{5}, 0.1,0.6\right\rangle\right\}, \\
& B_{2}=\left\{\left\langle C_{1}, 0.0,0.8\right\rangle,\left\langle C_{2}, 0.4,0.4\right\rangle,\left\langle C_{3}, 0.6,0.1\right\rangle,\left\langle C_{4}, 0.1,0.7\right\rangle,\left\langle C_{5}, 0.1,0.8\right\rangle\right\}, \\
& B_{3}=\left\{\left\langle C_{1}, 0.8,0.1\right\rangle,\left\langle C_{2}, 0.8,0.1\right\rangle,\left\langle C_{3}, 0.0,0.6\right\rangle,\left\langle C_{4}, 0.2,0.7\right\rangle,\left\langle C_{5}, 0.0,0.5\right\rangle\right\}, \\
& B_{4}=\left\{\left\langle C_{1}, 0.6,0.1\right\rangle,\left\langle C_{2}, 0.5,0.4\right\rangle,\left\langle C_{3}, 0.3,0.4\right\rangle,\left\langle C_{4}, 0.7,0.2\right\rangle,\left\langle C_{5}, 0.3,0.4\right\rangle\right\} .
\end{aligned}
$$

Then, we utilize the improved correlation coefficient to derive a diagnosis for each patient $B_{j}(j=1,2,3,4)$. Let $c=3$ in Formula (4). Then, all the diagnosis results for the considered patients are listed in Table 3.

Finally, from the arguments in Table 3, we derive a proper diagnosis as follows:

Al developed malaria; Bob had some problems with his stomach; Joe got typhoid; Ted got viral fever.

Based on the correlation coefficient (1), Xu's diagnosis results [19] (Al suffered from malaria, Bob from a stomach problem, and both Joe and Ted from viral fever) are different from the results obtained by the improved correlation coefficient (4). Because the improved correlation coefficient has a wider scope of application and can solve the problems of the existing correlation coefficient (mentioned in Remark 2), the diagnosis results obtained by the improved correlation coefficient (4) are more reasonable. Furthermore, Vlachos and Sergiadis [16] made diagnosis utilizing the symmetric discrimination information measure $D_{I F S}(A, B)$ and pointed out that the diagnosis results of $D_{I F S}(A, B)$ were more effective than the results of distance-based methods and the similarity-dissimilarity measure. The diagnosis results obtained by $D_{I F S}(A, B)$ were as follows: Al got viral fever, Bob had some problems with his stomach, Joe got typhoid, and Ted got viral fever, which are also different from those by the improved correlation coefficient (4). The differences are because that the results derived using $D_{I F S}(A, B)$ are prone to the influence of unfair arguments with too high or too low values, while the improved correlation coefficient (4) can relieve the influence of these unfair arguments by emphasizing the role of the considered arguments as a whole [21].

Table 3: Correlation Coefficients of Symptoms for Each Patient.

\begin{tabular}{rrrrrr}
\hline & $A_{1}$ & $A_{2}$ & $A_{3}$ & $A_{4}$ & $A_{5}$ \\
\hline$B_{1}$ & 0.7727 & 0.7860 & 0.7557 & 0.4740 & 0.4697 \\
$B_{2}$ & 0.6136 & 0.4717 & 0.6896 & 0.8848 & 0.5828 \\
$B_{3}$ & 0.7132 & 0.6221 & 0.7713 & 0.5296 & 0.4805 \\
$B_{4}$ & 0.7864 & 0.7227 & 0.6747 & 0.5600 & 0.4987 \\
\hline
\end{tabular}




\subsection{The Application in Clustering}

In the following, we will show that the improved correlation coefficient (4) is more effective than correlation coefficients (1) and (2) in clustering.

For the object sets $A_{i}(i=1,2, \ldots, m)$, suppose that the weight vector of $x_{i}(i=1,2, \ldots, n)$ is $\omega=\left(\omega_{1}, \omega_{2}, \ldots, \omega_{n}\right)^{\mathrm{T}}$, then we discuss the clustering steps:

- Step 1: Utilize Eq. (4) to calculate the correlation coefficient $\rho_{1}^{*}\left(A_{i}, A_{j}\right)$ between object sets $A_{i}$ and $A_{j}$ $(i, j=1,2, \ldots, m)$, and construct association matrix $\dot{C}=\left(\dot{\rho}_{i j}\right)_{m \times m}$, where $\dot{\rho}_{i j}=\rho_{1}^{*}\left(A_{i}, A_{j}\right) \quad(i, j=1,2, \cdots, m)$.

- Step 2: Obtain equivalence association matrix $\overline{\dot{C}}=\left(\bar{\rho}_{i j}\right)_{m \times m}$ by finite times compositions of $\dot{C}=\left(\dot{\rho}_{i j}\right)_{m \times m}$ and construct $\lambda$-cutting matrix $\overline{\dot{C}}_{\lambda}=\left(\lambda \bar{\rho}_{i j}\right)_{m \times n}$ of the equivalence association matrix $\overline{\dot{C}}$.

- Step 3: For the $\lambda$-cutting matrix $\overline{\dot{C}}_{\lambda}$, if all the elements of the $i$ th row or column are the same with the jth row or column, we assert object sets $A_{i}$ and $A_{j}$ are in the same class.

Example 4 ([28]): A car market is going to classify five different cars. Every car has six evaluation factors: (1) $G_{1}$ - fuel consumption; (2) $G_{2}$ - degree of friction; (3) $G_{3}$ - the price; (4) $G_{4}$ - degree of comfort; (5) $G_{5}$ design; (6) $G_{6}$ - security. The information of every car under each evaluation factor is represented by intuitionistic fuzzy numbers, which are shown in Table 4.

If the weight vector of the element $x_{i} \quad(i=1,2, \ldots, 6)$ is $\omega=\left(\frac{1}{6}, \frac{1}{6}, \cdots, \frac{1}{6}\right)^{\mathrm{T}}$, let $c=3$, then we can classify the cars by the improved correlation coefficient $\rho_{1}^{*}\left(A_{i}, A_{j}\right)$.

First, we utilize the improved correlation coefficient $\rho_{1}^{*}\left(A_{i}, A_{j}\right)$ to calculate the correlation between each pair of IFSs $A_{i}$ and $A_{j} \quad(i, j=1,2, \ldots, 5)$, and construct an association matrix:

$$
C=\left[\begin{array}{ccccc}
1 & 0.8422 & 0.8203 & 0.7981 & 0.6885 \\
0.8422 & 1 & 0.8634 & 0.8109 & 0.8096 \\
0.8203 & 0.8634 & 1 & 0.8047 & 0.7535 \\
0.7981 & 0.8109 & 0.8047 & 1 & 0.7073 \\
0.6885 & 0.8096 & 0.7535 & 0.7073 & 1
\end{array}\right]
$$

Then, we obtain an equivalent association matrix by finite times compositions of $C$. For

$$
C^{2}=\left[\begin{array}{ccccc}
1 & 0.8422 & 0.8422 & 0.8109 & 0.8096 \\
0.8422 & 1 & 0.8634 & 0.8109 & 0.8096 \\
0.8422 & 0.8634 & 1 & 0.8109 & 0.8096 \\
0.8109 & 0.8109 & 0.8109 & 1 & 0.8096 \\
0.8096 & 0.8096 & 0.8096 & 0.8096 & 1
\end{array}\right] \nsubseteq \subset C
$$

Table 4: Information.

\begin{tabular}{rrrrrrr}
\hline & $\boldsymbol{x}_{1}$ & $\boldsymbol{x}_{1}$ & $\boldsymbol{x}_{3}$ & $\boldsymbol{x}_{4}$ & $\boldsymbol{x}_{5}$ & $\boldsymbol{x}_{6}$ \\
\hline$A_{1}$ & $(0.3,0.5)$ & $(0.6,0.1)$ & $(0.4,0.3)$ & $(0.8,0.1)$ & $(0.1,0.6)$ & $(0.5,0.4)$ \\
$A_{2}$ & $(0.6,0.3)$ & $(0.5,0.2)$ & $(0.6,0.1)$ & $(0.7,0.1)$ & $(0.3,0.6)$ & $(0.4,0.3)$ \\
$A_{3}$ & $(0.4,0.4)$ & $(0.8,0.1)$ & $(0.5,0.1)$ & $(0.6,0.2)$ & $(0.4,0.5)$ & $(0.3,0.2)$ \\
$A_{4}$ & $(0.2,0.4)$ & $(0.4,0.1)$ & $(0.9,0.0)$ & $(0.8,0.1)$ & $(0.2,0.5)$ & $(0.7,0.1)$ \\
$A_{5}$ & $(0.5,0.2)$ & $(0.3,0.6)$ & $(0.6,0.3)$ & $(0.7,0.1)$ & $(0.6,0.2)$ & $(0.5,0.3)$ \\
\hline
\end{tabular}




$$
C^{4}=\left[\begin{array}{ccccc}
1 & 0.8422 & 0.8422 & 0.8109 & 0.8096 \\
0.8422 & 1 & 0.8634 & 0.8109 & 0.8096 \\
0.8422 & 0.8634 & 1 & 0.8109 & 0.8096 \\
0.8109 & 0.8109 & 0.8109 & 1 & 0.8096 \\
0.8096 & 0.8096 & 0.8096 & 0.8096 & 1
\end{array}\right]=C^{2}
$$

we get the equivalent association matrix $C^{2}$, denoted by $\tilde{C}$.

Finally, due to the fact that the confidence level $\lambda$ has a close relationship with the elements of the equivalent association matrix $\tilde{C}$, we give a detailed sensitivity analysis with respect to the confidence level $\lambda$ and get all the possible classifications of the five cars $A_{i}(i=1,2,3,4,5)$.

1. if $0 \leq \lambda \leq 0.8096, \quad \tilde{C}_{\lambda}=\left[\begin{array}{lllll}1 & 1 & 1 & 1 & 1 \\ 1 & 1 & 1 & 1 & 1 \\ 1 & 1 & 1 & 1 & 1 \\ 1 & 1 & 1 & 1 & 1 \\ 1 & 1 & 1 & 1 & 1\end{array}\right]$

then the cars are of the same type: $\left\{A_{1}, A_{2}, \ldots, A_{5}\right\}$;

2. if $0.8096<\lambda \leq 0.8109, \quad \tilde{C}_{\lambda}=\left[\begin{array}{lllll}1 & 1 & 1 & 1 & 0 \\ 1 & 1 & 1 & 1 & 0 \\ 1 & 1 & 1 & 1 & 0 \\ 1 & 1 & 1 & 1 & 0 \\ 0 & 0 & 0 & 0 & 1\end{array}\right]$

then the cars are classified into the following two types: $\left\{A_{1}, A_{2}, A_{3}, A_{4}\right\},\left\{A_{5}\right\}$;

3. if $0.8109<\lambda \leq 0.8422, \quad \tilde{C}_{\lambda}=\left[\begin{array}{ccccc}1 & 1 & 1 & 0 & 0 \\ 1 & 1 & 1 & 0 & 0 \\ 1 & 1 & 1 & 0 & 0 \\ 0 & 0 & 0 & 1 & 0 \\ 0 & 0 & 0 & 0 & 1\end{array}\right]$

then the cars are classified into the following three types: $\left\{A_{1}, A_{2}, A_{3}\right\},\left\{A_{4}\right\},\left\{A_{5}\right\}$;

4. if $0.8422<\lambda \leq 0.8634, \quad \tilde{C}_{\lambda}=\left[\begin{array}{ccccc}1 & 0 & 0 & 0 & 0 \\ 0 & 1 & 1 & 0 & 0 \\ 0 & 1 & 1 & 0 & 0 \\ 0 & 0 & 0 & 1 & 0 \\ 0 & 0 & 0 & 0 & 1\end{array}\right]$

then the cars are classified into the following four types: $\left\{A_{1}\right\},\left\{A_{2}, A_{3}\right\},\left\{A_{4}\right\},\left\{A_{5}\right\}$; 
5. if $0.8634<\lambda \leq 1, \quad \tilde{C}_{\lambda}=\left[\begin{array}{lllll}1 & 0 & 0 & 0 & 0 \\ 0 & 1 & 0 & 0 & 0 \\ 0 & 0 & 1 & 0 & 0 \\ 0 & 0 & 0 & 1 & 0 \\ 0 & 0 & 0 & 0 & 1\end{array}\right]$

then the cars are classified into the following five types: $\left\{A_{1}\right\},\left\{A_{2}\right\},\left\{A_{3}\right\},\left\{A_{4}\right\},\left\{A_{5}\right\}$.

From the above analysis, we know that the improved correlation coefficient (4) can be applied in the clustering of IFSs effectively. Moreover, there are five situations based on the association matrix, which is constructed by the improved correlation coefficient (4), while only three situations were obtained in Ref. [28]. It can be seen that the improved correlation coefficient has higher accuracy in clustering.

\section{Conclusion}

The existing correlation coefficients did not meet some desirable properties in the IFS theory. In order to solve this problem, we have introduced an improved correlation coefficient of the IFSs. We have shown the advantages of the improved correlation coefficient by comparing with the correlation coefficients proposed by $\mathrm{Xu}[19,21]$. Then, we have further generalized it to the IVIFS environment. Finally, the improved correlation coefficient has also been applied effectively in medical diagnosis and clustering.

Acknowledgments: This work was supported by the National Natural Science Foundation of China (11571158).

\section{Bibliography}

[1] K. Atanassov, Intuitionistic fuzzy sets, Fuzzy Sets Syst. 20 (1986), 87-96.

[2] K. Atanassov and G. Gargov, Interval-valued intuitionistic fuzzy sets, Fuzzy Sets Syst. 31 (1989), 343-349.

[3] N. Chen, Z. S. Xu and M. M. Xia, Correlation coefficients of hesitant fuzzy sets and their applications to clustering analysis, Appl. Math. Model. 37 (2013), 2197-2211.

[4] C. H. Chu, K. C. Hung and P. Julian, A complete pattern recognition approach under Atanassov's intuitionistic fuzzy sets, Knowl. Based Syst. 66 (2014), 36-45.

[5] G. Deschrijver and E. E. Kerre, On the relationship between some extensions of fuzzy set theory, Fuzzy Sets Syst. 133 (2003), 227-235.

[6] A. Fiche, J. Cexus, A. Martin and A. Khenchaf, Features modeling with an $\alpha$-stable distribution: application to pattern recognition based on continuous belief functions, Inf. Fusion 14 (2013), 504-520.

[7] J. Goguen, L-fuzzy sets, J. Math. Anal. Appl. 18 (1967), 145-174.

[8] C. Grosan, A. Abraham and S. Tigan, Multicriteria programming in medical diagnosis and treatments, Appl. Soft Comput. 8 (2008), 1407-1417.

[9] H. L. Huang, (T,S)-Based interval-valued intuitionistic fuzzy composition matrix and its application for clustering, Iran. J. Fuzzy Syst. 9 (2012), 7-19.

[10] W. L. Hung, Using statistical viewpoint in developing correlation of intuitionistic fuzzy sets, Int. J. Uncertain. Fuzziness Knowl. Based Syst. 9 (2001), 509-516.

[11] W. L. Hung and J. W. Wu, Correlation of intuitionistic fuzzy sets by centroid method, Inf. Sci. 144 (2002), 219-225.

[12] H. Laanaya, A. Martin, D. Aboutajdine and A. Khenchaf, Support vector regression of membership functions and belief functions - application for pattern recognition, Inf. Fusion 11 (2010), 338-350.

[13] M. Z. Ranitović, Lattice representations of interval-valued fuzzy sets, Fuzzy Sets Syst. 236 (2014), 50-57.

[14] E. Szmidt and J. Kacprzyk, A similarity measure for intuitionistic fuzzy sets and its application in supporting medical diagnostic reasoning, Lect. Notes Artif. Intell. 3070 (2004), 388-393.

[15] Z. Takáč, Inclusion and subsethood measure for interval-valued fuzzy sets and for continuous type-2 fuzzy sets, Fuzzy Sets Syst. 224 (2013), 106-120. 
[16] I. K. Vlachos and D. D. Sergiadis, Intuitionistic fuzzy information-application to pattern recognition, Pattern Recognit. Lett. 28 (2007), 197-206.

[17] P. Z. Wang, Fuzzy set theory and applications, Shanghai Scientific and Technical Publishers, Shanghai, 1983.

[18] G. J. Wang and Y. Y. He, Intuitionistic fuzzy sets and L-fuzzy sets, Fuzzy Sets Syst. 110 (2000), 271-274.

[19] Z. S. Xu, On correlation measures of intuitionistic fuzzy sets, Lect. Notes Comput. Sci. 4224 (2006), 16-24.

[20] Z. S. Xu, Hesitant fuzzy multi-attribute decision making based on TOPSIS with incomplete weight information, Knowl. Based Syst. 52 (2013), 53-64.

[21] Z. S. Xu and X. Q. Cai, Intuitionistic fuzzy information aggregation: theory and applications, Springer, Heidelberg, 2012.

[22] Z. S. Xu, J. Chen and J. J. Wu, Clustering algorithm for intuitionistic fuzzy sets, Inf. Sci. 19 (2008), 3775-3790.

[23] W. Yao, On many-valued stratified L-fuzzy convergence spaces, Fuzzy Sets Syst. 159 (2008), 2503-2519.

[24] Z. L. Yue, Group decision making with multi-attribute interval data, Inf. Fusion 14 (2013), 551-561.

[25] S. H. Yue, T. Wu, J. Pan and H. Wang, Fuzzy clustering based ET image fusion, Inf. Fusion 14 (2013), 487-497.

[26] L. A. Zadeh, Fuzzy sets, Inf. Control 8 (1965), 338-353.

[27] L. A. Zadeh, Outline of a new approach to the analyses of complex systems and decision processes interval-valued fuzzy sets, IEEE Trans. Syst. Man Cybern. 3 (1973), 28-44.

[28] H. M. Zhang, Z. S. Xu and Q. Chen, Clustering method of intuitionistic fuzzy sets, Control Decis. 22 (2007), 882-888 (in Chinese).

[29] H. Y. Zhang, W. X. Zhang and C. L. Mei, Entropy of interval-valued fuzzy sets based on distance and its relationship with similarity measure, Knowl. Based Syst. 22 (2009), 449-454.

[30] Z. H. Zhang, J. Y. Yang, Y. P. Ye and Q. Zhang, A type of score function on intuitionistic fuzzy sets with double parameters and its application to pattern recognition and medical diagnosis, Procedia Eng. 29 (2012), 4336-4342. 